\title{
Autismo e mães com e sem estresse: análise da sobrecarga materna e do suporte familiar
}

\author{
Kátia Carvalho Amaral Faro ${ }^{1}$ \\ Rosita Barral Santos $\mathbb{( i}^{2}$ \\ Cleonice Alves Bosa ${ }^{1}{ }^{1}$ \\ Adriana Wagner (1) ${ }^{1}$ \\ Simone Souza da Costa Silva $\oplus^{3}$ \\ ${ }^{1}$ Universidade Federal do Rio Grande do Sul, RS, Brasil. \\ ${ }^{2}$ Universidade Federal da Bahia, BA, Brasil. \\ ${ }^{3}$ Universidade Federal do Pará, PA, Brasil.
}

\begin{abstract}
Resumo
O objetivo deste estudo foi comparar dois grupos de mães de crianças com autismo (com e sem estresse), quanto à: (a) sobrecarga de cuidado; (b) autonomia da criança; e (c) percepção de suporte familiar. Participaram 30 mães de crianças, de três a sete anos de idade, com Transtorno do Espectro Autista (TEA). Os instrumentos utilizados foram: Inventário Biosociodemográfico, Inventário de Sintoma de Stress de Lipp, Escala de Sobrecarga de Zarit, Inventário de Avaliação Pediátrica de Incapacidade e Inventário de Percepção de Suporte Familiar. Os resultados revelaram que, mães com estresse tiveram quase o dobro de percepção de sobrecarga, enquanto as sem estresse perceberam maior suporte familiar, principalmente nos aspectos de afetividade e autonomia em relação aos familiares, como expressão e comunicação de afetos e respeito pela sua liberdade e tomadas de decisões. Nesse sentido, os resultados apontaram a importância do suporte familiar na adaptação de mães de crianças com autismo.
\end{abstract}

Palavras-chave: estresse, maternidade, autismo, cuidador, relações familiares.

\section{Autism and mothers with and without stress: analysis of maternal burden and familiar support}

\begin{abstract}
The aim of this study was to compare mothers of autistic children, with or without stress regarding the burden of care, child's autonomy and perception of family support. Participants were 30 mothers of children with autism spectrum disorder (ASD) between 3 and 7 years old. The instruments used were: Inventário Biosociodemográfico, Inventário de Sintoma de Stress de Lipp, Escala de Sobrecarga de Zarit, Inventário de Avaliação Pediátrica de Incapacidade e Inventário de Percepção de Suporte Familiar. The results showed that mothers with stress had almost twice the perception of burden, whereas those without stress perceived themselves with greater family support, mainly regarding affect and autonomy in relation to their relatives, as expression and communication of affections and respect for their freedom and taken of decisions. Thus, the results pointed to the importance of family support for the maternal adaptation to their child's condition.
\end{abstract}

Keywords: stress, motherhood, autism, caregivers, family relations.

\section{Autismo y madres con y sin estrés: análisis de la sobrecarga materna y de lo soporte familiar}

\section{Resumen}

El objetivo de este estudio fue comparar a dos grupos de madres que tienen hijos autistas (con y sin estrés), en relación a (a) sobrecarga de cuidado, (b) autonomía de los niños y (c) percepción del soporte familiar. Han participado 30 madres de niños, de trés hasta los siete años de edad, con trastorno del espectro autista (TEA). Los instrumentos utilizados fueron: Inventário Biosociodemográfico, Inventário de Sintoma de Stress de Lipp, Escala de Sobrecarga de Zarit, Inventário de Avaliação Pediátrica de Incapacidade and Inventário de Percepção de Suporte Familiar. Los resultados han revelado que las madres con estrés tuvieron casi el doble de percepción de sobrecarga, mientras las sin estrés percibieron mayor soporte familiar, principalmente en los aspectos de afectividad y autonomía en relación a los familiares, como expresión y comunicación de afectos y respecto por su libertad y tomas de decisión. En ese aspecto, los resultados apuntan a la importancia del soporte familiar en la adaptación de madres de niños con autismo.

Palabras clave: estrés, maternidad, autismo, cuidador, relaciones familiares. 
O Transtorno do Espectro Autista (TEA) consiste em alterações no neurodesenvolvimento ainda no início da infância, cujas características principais são déficits clinicamente significativos e persistentes na comunicação e interação social, associados à presença de padrões comportamentais restritos, inflexíveis e repetitivos (American Psychiatric Association [APA], 2013). Essas alterações envolvem: (a) dificuldades em desenvolver e iniciar interações espontâneas com pares; (b) ausência de reciprocidade social; (c) déficits expressivos na comunicação não verbal e verbal usadas para interação social; e (d) padrões atípicos restritos e repetitivos de comportamentos, interesses e atividades, tais como maneirismos motores e/ou verbais, hiper ou hiporreatividade a estímulos sensoriais, incluindo desorganização emocional e sensorial na quebra de rotinas (APA, 2013).

As dificuldades agravam-se quando esses fatores são associados a prejuízos cognitivos (APA, 2013). Nesses casos, sabe-se que há um baixo funcionamento intelectual e adaptativo que impactam em comportamentos indesejados, como a dificuldade de lidar com situações novas, a agressividade, a autoestimulação e o baixo desempenho escolar (Fombonne, 2009). A presença dessas condições pode aumentar o nível de dependência para atividades da vida diária e a diminuição das chances de inserção escolar e no trabalho (Fodstad \& Matson, 2008).

Embora a variabilidade das características do TEA possa comprometer mais algumas crianças do que outras, tendo em vista o caráter multifatorial do transtorno, ter um membro familiar com TEA provoca um impacto significativo na rotina e nas relações familiares (Fávero \& Santos, 2005; Gorlin, McAlpine, Garwick, \& Wieling, 2016; Meimes, Saldanha, \& Bosa, 2015; Pisula, 2011; Schmidt, Dell'Aglio, \& Bosa, 2007). Dillenburger, Keenan, Doherty, Byrne e Gallagher (2010) entrevistaram pais de crianças com TEA e profissionais da área de saúde com intuito de analisar semelhanças e diferenças nas visões sobre as dificuldades nos comportamentos infantis, o impacto da convivência com essas crianças na vida familiar e o apoio geral dado às famílias. Os autores identificaram que, mais de $90 \%$ dos pais relataram como suas maiores dificuldades a falta ou o prejuízo na interação, na comunicação e na linguagem. Além disso, tanto a família quanto os profissionais percebiam a preponderância do impacto do TEA na restrição da família em atividades sociais, lazer e viagens (Dillenburger et al., 2010).

Nesse sentido, vários estudos mencionam as demandas de cuidados intensivos diante das peculiaridades do transtorno que podem influenciar na adaptação familiar, levando algumas famílias a pas- sarem por: (a) problemas conjugais (Meimes et al., 2015); (b) percepção de sobrecarga de um dos membros (Misquiatti, Brito, Ferreira, \& Assumpção Junior, 2015); (c) estresse parental (Dabrowska \& Pisula, 2010); (d) impacto nos irmãos (Cezar \& Smeha, 2016; Gomes \& Bosa, 2004); e (e) dificuldades financeiras e isolamento (Dillenburger et al., 2010). Esses podem se caracterizar como fatores de risco para o adoecimento físico e prejuízos na saúde mental.

A perspectiva sistêmica considera que as condições próprias do TEA podem causar um desequilíbrio familiar por afetar, principalmente, os processos de comunicação e a reciprocidade das relações interpessoais (Bosa, Sifuentes, \& Semensato, 2012). A família, nesse contexto, sofre alterações no seu funcionamento no que diz respeito ao desempenho dos papéis, pois é demandada pela condição da criança, que requer interações diferenciadas conforme o seu estágio de desenvolvimento. Nos casos mais graves do TEA, o impacto na família tende a ser maior, pois há uma necessidade de cuidado integral com a criança devido às dificuldades no controle dos impulsos, aos prejuízos no julgamento de perigo e em alguns casos, comportamentos autolesivos (Gorlin et al., 2016).

Ressalta-se, ainda, que cada membro da família se adapta de forma particular aos estressores advindos dessa interação, conforme o papel que desempenha (Rolland, 2001). Dessa forma, considera-se que a dinâmica de funcionamento dos processos familiares na resolução de problemas contribui de maneira significativa tanto para a adaptação positiva quanto para a disfunção individual e relacional (Walsh, 2016b). A adaptação, ou a forma utilizada pela família para se auto-organizar diante das transições vividas, tem duas dimensões primordiais que favorecem a recuperação em contextos de transição ou crise: (a) a flexibilidade, ou a abertura para a mudança e o enfrentamento dos novos desafios; e (b) a estabilidade, garantida pela continuidade, confiança e previsibilidade em meio à turbulência (Walsh, 2006a).

Dentre os familiares cujo impacto e as demandas de cuidado são reportadas na literatura com maior frequência e intensidade, mães de crianças com autismo são amplamente identificadas como quem mais sofre física e mentalmente diante do cuidado intensivo (García-Lopez, Sarriá, \& Pozo, 2016; Pisula, 2011; Pozo \& Sarriá, 2014). Estudos que comparam mães de crianças com TEA com mães de crianças com outras alterações no desenvolvimento, como deficiência intelectual ou mesmo desenvolvimento típico, têm apontado diferenças em medidas de estresse e nas estratégias de enfrentamento, apresentando maiores escores nas avaliações de estresse (Dabrowska \& 
Pisula, 2010; Estes et al., 2009; Tomanik, Harris, \& Hawkins, 2004). Essa diferença também foi identificada entre os pais e as mães de crianças com autismo, com mães apresentando escores maiores de estresse do que os pais (Dabrowska \& Pisula, 2010; García-Lopez et al., 2016). Em geral, nesses contextos familiares, a mãe se constitui como a principal cuidadora dessas crianças (García-Lopez et al., 2016; Zaidman-Zait et al., 2017).

$\mathrm{O}$ estresse e a sobrecarga, nesse sentido, têm sido medidas recorrentes em várias pesquisas com intuito de avaliar o impacto do cuidado nessas mães. As características da criança que influenciam o estresse materno são relacionadas à maior dificuldade de comunicação, autocuidado e problemas comportamentais, como agitação, irritação ou desobediência, mesmo em estudos que comparam crianças com TEA e outras condições (Hastings et al., 2005; Tomanik et al., 2004). Tomanik et al. (2004), por exemplo, em um estudo que comparou uma amostra de crianças com TEA e com atraso desenvolvimental sem autismo (DD), encontraram maiores níveis de problemas comportamentais e menores níveis de habilidades de vida diária influenciando o estresse materno no grupo de TEA em relação ao grupo de mães de crianças DD.

Assim sendo, o estresse caracteriza-se como uma reação psicofisiológica adaptativa diante de demandas percebidas pelo indivíduo como excedentes aos recursos que dispõe no momento, sejam recursos internos ou externos (Faro \& Pereira, 2013; Lazarus \& Folkman, 1984), portanto, é intrinsecamente ligado à percepção cognitiva da experiência estressante (Lazarus \& Folkman, 1984). Em mães de crianças com TEA, além das características da criança e seus recursos psicológicos, outras características do contexto estão associadas à presença de estresse. Phetrasuwan e Miles (2009), em um estudo correlacional em que foram investigadas as fontes estressoras e a sua relação com estresse, bem-estar e depressão, identificaram que mães com níveis menores de educação e com menor renda apresentaram maiores níveis de estresse.

Outro fator que estudiosos têm relacionado ao estresse materno é a sobrecarga (Green \& Carter, 2014; Pozo \& Sarriá, 2014; Schmidt \& Bosa, 2003; ZaidmanZait et al., 2017), conceito que trata do impacto negativo em um ou mais membros da família, devido à responsabilidade de realizar tarefas de cuidado a outro membro familiar, tais como filhos, pais e irmãos (Pereira \& Soares, 2011). Esses autores acrescentam que o impacto do cuidado intensivo leva o familiar a não priorizar suas necessidades e desejos, o que gera efeitos de natureza objetiva (e.g. dificuldades financeiras, de saúde, profissionais) e subjetiva (percepção sobre a situação e questões afetivas) no cuidador.
Em outra pesquisa com familiares de crianças com TEA acerca das suas percepções a respeito da sobrecarga, observou-se que $85 \%$ dos cuidadores responsáveis por assistir as crianças eram do gênero feminino e $80 \%$ eram mães (Misquiatti et al., 2015). Os resultados apontaram para consequências e limitações na vida pessoal do cuidador, tais como diminuição do tempo disponível, consequências negativas para a saúde e, ainda, a necessidade de alteração de diversos hábitos para ter condições de atender às demandas do familiar (Misquiatti et al., 2015).

Assim, uma variável frequentemente relatada como atenuante do estresse e da sobrecarga é o suporte social, e de modo mais específico o suporte familiar. Esse é definido pela percepção do indivíduo sobre a disponibilidade e o apoio de sua família, considerado essencial para um bom desenvolvimento do indivíduo (Baptista, 2009), fundamental para o equilíbrio das famílias de crianças com autismo (Schmidt \& Bosa, 2003). A perspectiva sistêmica avalia o suporte familiar de forma multidimensional, incluindo aspectos como clareza da comunicação, proximidade versus distanciamento entre os membros, organização do grupo em contextos específicos, afetividade, o desempenho dos papéis familiares e as crenças sobre a representação desses papéis (Hill, Fonagy, Safier, \& Sargent, 2003).

Há apontamentos na literatura sobre a necessidade de apoio social reportada por mães de crianças com autismo, em particular o suporte familiar, sendo percebido como a principal fonte de auxílio para elas nas adversidades que surgem em decorrência das demandas do filho com autismo (Hartley \& Schultz, 2015; Smeha \& Cezar, 2011). Um estudo realizado com 283 mães de crianças com TEA buscou examinar associações longitudinais entre problemas de comportamento infantil, estratégias de enfrentamento, recursos sociais e estresse parental. Desde a identificação de TEA até dois anos após o período do diagnóstico, os resultados apontaram para a diminuição do estresse parental em mães que tiveram níveis altos ou crescentes de apoio familiar, enquanto níveis altos ou crescentes de disfunção familiar foram associados ao aumento do estresse (Zaidman-Zait et al., 2017).

Assim, as evidências encontradas nesse levantamento da literatura mostram que uma criança com TEA demanda da família a adaptação frente às necessidades e dificuldades suscitadas pelas características desse transtorno. Esse fenômeno é relatado como potencialmente estressor da família, especialmente das mães, reportadas como as principais cuidadoras dessas crianças. Nesse contexto, as demandas de cuidado relacionadas ao grau de autonomia da criança, quando não equilibradas nas divisões de tarefas da família, 
podem favorecer a sobrecarga de atividades de cuidado da mãe. Somam-se a esse fato aspectos cognitivos que influenciam a percepção de eventos como estressantes e que podem levar a condições físicas e psicológicas de estresse. Em contrapartida, perceber a família como um contexto suportivo aparece associado à ausência ou menores índices de estresse.

Portanto, embora haja fartas evidências sobre a sobrecarga materna em famílias de crianças com autismo (Gomes, Lima, Bueno, Araújo, \& Souza, 2015; Macedo, Silva, Paiva, \& Ramos, 2015; Meimes et al., 2015), menos atenção é dada à investigação dos fatores que de fato contribuem para a mitigação do impacto do estresse. Torna-se relevante investigar quais variáveis favorecem a redução do estresse em mães de crianças com TEA inseridas em contextos socioculturais diversos, levando em consideração a percepção das mães diante desse fenômeno. Dessa forma, os resultados de investigações dessa natureza podem contribuir para futuras intervenções voltadas para o auxílio na adaptação da família ao contexto do TEA, e para a minimização dos potenciais estressores e maximização dos recursos saudáveis que a família dispõe no enfrentamento da situação adversa.

Diante do exposto, o objetivo deste estudo foi comparar mães de crianças com autismo, com e sem estresse, quanto à sobrecarga de cuidado, à autonomia da criança e à percepção de suporte familiar. As hipóteses do estudo são de que: (a) filhos de mães com estresse possuem maior prejuízo na autonomia; e (b) mães com estresse apresentam maior percepção de sobrecarga e menor percepção de suporte familiar.

\section{Método}

\section{Delineamento e participantes}

A pesquisa foi de natureza quantitativa e descritiva, da qual participaram 30 mulheres, mães de crianças com autismo da cidade de Belém-PA, oriundas de um banco de dados da pesquisa de Amaral (2013) ${ }^{1}$. O delineamento utilizado foi o de comparação de grupos, sendo que as 30 mães foram alocadas em dois grupos constituídos por mães com estresse $(\mathrm{GCE} ; n=21)$ e sem estresse (GSE; $n=9)$. A alocação foi realizada com base na classificação do Inventário de Sintomas de Stress para Adultos Lipp (ISSL; Lipp, 2000, descrita na seção de Instrumentos).

A seleção das participantes deu-se por meio de uma amostragem não probabilística por conveniência, cujo critério de inclusão era ser mãe de criança com

\footnotetext{
${ }^{1}$ Projeto aprovado pelo do Comitê de Ética do Núcleo de Medicina Tropical da UFPA sob o no 275.476/2013, atendendo à Resolução de no 466/2012 do Conselho Nacional de Saúde.
}

idade entre três e sete anos, com diagnóstico de TEA realizado por médicos especializados e com experiência nessa área. $\mathrm{O}$ critério de exclusão foi o de a criança não apresentar outras condições médicas ou genéticas associadas, como outros transtornos do desenvolvimento, deficiências sensoriais ou motoras, identificadas a partir dos registros clínicos.

\section{Instrumentos}

Inventário Biosociodemográfico. O inventário desenvolvido para o estudo de Amaral (2013) buscou obter informações pessoais a respeito da mãe e da criança com itens de identificação, composição familiar, renda, ocupação parental e informações referentes aos tratamentos da criança. No inventário foi realizado um levantamento de percepção de estressores em relação às características de TEA da criança (e. g. prejuízos na interação social, problemas no sono, ecolalia, etc.), em que a mãe identificava, em uma lista, a presença e quais características de seu filho ela vivenciava e considerava como um estressor.

Inventário de Sintomas de Stress para Adultos de Lipp - ISSL. Este inventário tem por finalidade identificar de forma objetiva a ausência ou presença da sintomatologia de estresse, bem como o tipo de sintoma (se somático ou psicológico) e em qual fase se encontra (alerta, resistência, quase exaustão e exaustão) (Lipp, 2000). O instrumento é composto por uma lista de 37 itens de sintomas físicos e 19 itens de sintomas psicológicos, dispostos em três quadros de tempo: nas últimas 24 horas, na última semana e no último mês. A sequência dos itens segue uma ordem de intensidade dos sintomas, que caracterizam as fases do stress anteriormente citadas. Os pontos de corte utilizados para discriminar os grupos quanto à presença (nas quatro fases) ou ausência de estresse foram baseados nos escores normativos do instrumento.

Escala de sobrecarga (ES) - Burden Interview. Desenvolvida por Zarit, Reever e Bach-Peterson (1980) foi validada para o Brasil por Taub, Andreoli e Bertolucci (2004). Tem por principal objetivo avaliar a sobrecarga subjetiva dos familiares cuidadores de pacientes psiquiátricos. A ES avalia cinco aspectos da vida deste cuidador familiar: saúde, vida social e pessoal, situação financeira, bem-estar emocional e relações interpessoais em 22 itens. A pontuação se dá de 0 a 4, sendo 0 equivalente a pouca ou nenhuma e 4 a sempre.

Inventário de Avaliação Pediátrica de Incapacidade - PEDI. O Inventário de Avaliação Pediátrica de Incapacidade foi desenvolvido por Haley, Coster, Ludlow, Haltiwanger e Andrellos (1992) e adaptado para uma versão brasileira por Mancini (2005). O objetivo desse instrumento é fornecer uma descrição 
detalhada do desempenho funcional de crianças de seis meses a sete anos e meio de idade (ou se tendo alguma deficiência, ter idade funcional equivalente a menos que sete anos e meio) em ambiente doméstico, para fins de avaliação. Para este estudo, utilizou-se a subescala Assistência do adulto, administrada através de entrevista, para avaliar a quantidade de ajuda fornecida pelo cuidador de referência. Essa subescala é composta por 20 atividades funcionais nas áreas de autocuidado (oito itens), mobilidade (sete itens) e função social (cinco itens). Os itens são pontuados a partir de uma escala de cinco pontos, onde zero referese à assistência total do cuidador para realizar uma tarefa e cinco indica que a criança a desempenha de forma totalmente independente.

Inventário de Percepção de Suporte Familiar IPSF. O Inventário de Percepção de Suporte Familiar (Baptista, 2009) tem por objetivo avaliar o quanto a pessoa percebe sua família como suportiva no geral e também através de três construtos: afetividade, adaptação e autonomia. O instrumento é composto por 42 afirmações cuja resposta é determinada por uma escala de três pontos (quase nunca ou nunca/ às vezes/quase sempre ou sempre). Seus escores são classificados por baixo, médio-baixo, médio-alto e alto.

\section{Procedimentos}

As mães foram recrutadas do Núcleo de Atendimento Educacional Especializado aos Transtornos Globais do Desenvolvimento (NATEE) e de outras pesquisas sobre autismo do Núcleo de Teoria e Pesquisa do Comportamento da Universidade Federal do Pará (NTPC/UFPA). A coleta de dados foi realizada nos locais de preferência das mães, nas suas próprias residências ou nos locais de atendimento das crianças (durante o período de espera do término da sessão, em salas reservadas), com duração em torno de 1 h e 30 minutos.

As análises dos dados foram realizadas com estatísticas descritivas (frequências, médias e desvio padrão) e para as análises de comparação de grupos (nas variáveis sociodemográficas e principais) foi utilizado o teste não paramétrico de Mann-Whitney, com $\mathrm{p}<0,05$, através do software Statistical Package for the Social Sciences (SPSS) v.18.

\section{Resultados}

\section{Caracterização da amostra}

As características sociodemográficas das mães (idade, escolaridade, estado conjugal e ocupação)

TABELA 1

Características Sociodemográficas das Mães e das Crianças com TEA, Por Grupo

\begin{tabular}{|c|c|c|c|c|c|c|}
\hline \multirow{2}{*}{ Características } & \multicolumn{3}{|c|}{ Sem Estresse $(n=9)$} & \multicolumn{3}{|c|}{ Com Estresse $(n=21)$} \\
\hline & $n(f)$ & $M(S D)$ & Min:Máx & $n(f)$ & $M(S D)$ & Min:Máx \\
\hline \multicolumn{7}{|l|}{ Mães } \\
\hline Idade & & $36,6(4,4)$ & $29: 43$ & & $35,8(4,8)$ & $28: 45$ \\
\hline Renda* & & $8(5,7)$ & $2: 19$ & & $6,1(8,4)$ & 1: 40 \\
\hline \multicolumn{7}{|l|}{ Escolaridade } \\
\hline Pós-graduação & $3(33,3)$ & & & - & & \\
\hline Ensino superior & $2(22,2)$ & & & $11(52,4)$ & & \\
\hline Ensino médio & $4(44,4)$ & & & $9(42,9)$ & & \\
\hline Ensino fundamental & - & & & $1(4,8)$ & & \\
\hline \multicolumn{7}{|l|}{ Estado conjugal } \\
\hline União estável & $8(88,9)$ & & & $17(81)$ & & \\
\hline Separada & - & & & $4(19)$ & & \\
\hline Solteira & $1(11,1)$ & & & - & & \\
\hline \multicolumn{7}{|l|}{ Ocupação } \\
\hline Trabalhando & $7(77,8)$ & & & $13(61,9)$ & & \\
\hline Do lar & $2(22,2)$ & & & $8(38,1)$ & & \\
\hline \multicolumn{7}{|l|}{ Crianças } \\
\hline Idade (anos) & & $5,6(1,1)$ & $4: 7$ & & $5,2(1,2)$ & $3: 7$ \\
\hline Tempo de diagnóstico (anos) & & $2,9(1,1)$ & $1: 5$ & & $2,4(1,1)$ & $1: 5$ \\
\hline \multicolumn{7}{|l|}{ Sexo } \\
\hline Masculino & $6(66,7)$ & & & $18(85,7)$ & & \\
\hline Feminino & $3(33,3)$ & & & $3(14,3)$ & & \\
\hline \multicolumn{7}{|l|}{ Frequenta a escola } \\
\hline Sim & $8(88,9)$ & & & $18(85,7)$ & & \\
\hline Não & $1(11,1)$ & & & $3(14,3)$ & & \\
\hline
\end{tabular}

* Valores convertidos em salários mínimos. 
e das crianças (idade, tempo de diagnóstico, sexo e escolarização) podem ser observadas na Tabela 1. Não foram verificadas diferenças nessas variáveis entre os grupos de mãe com e sem estresse $(p<0,05)$.

Quanto às características familiares, a maioria da amostra de ambos os grupos apresentava uma estrutura familiar nuclear. No grupo de mães sem estresse (GSE), 77,8\% $(n=7)$ famílias se configuravam como nuclear e $22,2 \%(n=2)$ como estendidas. No grupo de mães com estresse (GCE), 57\% $(n=12)$ eram famílias nucleares, $28,6 \%(n=6)$ eram estendidas e $14 \%(n=3)$ se caracterizavam como monoparentais.

Foram investigadas questões referentes à participação das mães em grupos terapêuticos direcionados aos cuidadores de autismo e à satisfação com os atendimentos dos filhos. No GSE, 55,6\% $(n=5)$ reportaram participar ou já terem participado desse tipo de vivência, enquanto que no GCE a maioria das mães $62 \%(n=13)$ ainda não tinha participado de grupos voltados aos cuidadores/pais de pessoas com TEA. No que diz respeito à satisfação das mães sobre os atendimentos dos seus filhos, 88,9\% $(n=8)$ das mulheres do GSE se sentiam satisfeitas ou muito satisfeitas, semelhante ao GCE $(81 \%, n=17)$, com diferença entre grupos de $U=81.500, p=0,523$.

Em relação às características de TEA identificadas pelas mães, as principais condições apontadas como estressoras são referentes a prejuízos na comunicação (GSE: 77,8\%, $n=7$; GCE: 85,7\%, $n=18$ ) e à presença de comportamentos repetitivos (GSE: 44,4\%, $n=4$; GCE: $66,7 \%, n=14$ ). Ademais, também foram reportadas questões relacionadas à: (a) problemas na alimentação (GSE: $66,7 \%, n=6$; GCE: $62 \%, n=13$ ), (b) ausência de linguagem (GSE: $66,7 \%, n=6$; GCE: $71,4 \%, n=15$ ), (c) alterações sensoriais (GSE: 44,4\%, $n=4$; GCE: $66,7 \%, n=14$ ) e (d) dificuldades no autocuidado (GSE: $55,6 \%, n=5$; GCE: $47,6 \%, n=10$ ).

\section{Estresse, autonomia da criança, sobrecarga e percepção de suporte familiar}

Uma análise detalhada das características das mulheres pertencentes ao GCE foi realizada em relação ao nível de estresse e tipo de sintomas no ISSL (Lipp, 2000). Dessa subamostra, $76 \%(n=16)$ apresentaram estresse entre os níveis de resistência e $24 \%$ reportaram o nível de quase exaustão $(n=5)$, com predomínio nos sintomas psicológicos $(76 \%, n=16)$.

No que concerne à autonomia dos filhos, medido através da subescala Assistência do Cuidador do PEDI, as mães do GSE pontuaram que $55 \%$ das crianças $(n=5)$ apresentaram desempenho inferior ao escore normativo do instrumento no item de autocuidado, $11 \%(n=1)$ no item de mobilidade e $67 \%(n=6)$ no item de função social. As mães do GCE apresentaram resultados na mesma direção, com $62 \%(n=13), 38 \%$ $(n=8)$ e $81 \%(n=17)$, respectivamente. As diferenças encontradas nas médias entre os grupos não foram estatisticamente significativas, conforme é possível observar na Tabela 2.

Quanto à percepção de sobrecarga (ES), 55,6\% $(n=5)$ das mães do GSE reportaram sentir-se com pouca ou nenhuma carga, enquanto as demais mantiveram escores de nível leve ou carga moderada $(22,2 \%$, $n=2)$ e de moderada a carga pesada $(22,2 \%, n=2)$. Já no GCE, o nível de percepção predominante foi de sobrecarga leve ou moderada $(66,7 \%, n=14), 23 \%$ $(n=23,8)$ das mães tiveram escores de carga moderada a pesada e $9,5 \%(n=2)$ carga severa. Conforme pode ser observado na Tabela 2, as diferenças entre o GSE $(M=19,00)$ e GCE $(M=39,00)$ foram significativas para as ES. O grupo de mães com estresse apresentou

TABELA 2

Comparação Entre os Grupos Com e Sem Estresse em Autonomia da Criança, Sobrecarga e Percepção de Suporte Familiar

\begin{tabular}{|c|c|c|c|c|c|c|c|}
\hline \multirow{2}{*}{ Variáveis } & \multicolumn{2}{|c|}{ Sem Estresse $(n=9)$} & \multicolumn{2}{|c|}{ Com estresse $(n=21)$} & \multirow{2}{*}{$U$} & \multirow{2}{*}{$p$} & \multirow{2}{*}{$d^{*}$} \\
\hline & $M(D P)$ & Mnd & $M(D P)$ & Mnd & & & \\
\hline \multicolumn{8}{|l|}{ Características da criança } \\
\hline PEDI - autocuidado & $30,73(20,41)$ & 20,00 & $25,90(14,54)$ & 22,70 & 83,00 & 0,600 & 0,19 \\
\hline PEDI - Mobilidade & $38,04(13,64)$ & 37,10 & $37,67(21,60)$ & 45,20 & 84,00 & 0,632 & 0,01 \\
\hline PEDI - social & $20,75(13,64)$ & 10,00 & $20,96(20,36)$ & 10,00 & 86,00 & 0,656 & 0,17 \\
\hline Sobrecarga do Cuidador (ES) & $24,67(16,38)$ & 19,00 & $40,14(17,70)$ & 39,00 & 45,00 & $0,025^{*}$ & 0,90 \\
\hline \multicolumn{8}{|c|}{ Percepção de Suporte Familiar (IPSF) } \\
\hline Escore total & $71,00(12,04)$ & 78,00 & $57,9(17,7)$ & 62,00 & 48,00 & $0,035^{*}$ & 0,84 \\
\hline Autonomia & $14,67(2,18)$ & 16,00 & $11,00(4,8)$ & 16,00 & 45,00 & $0,021 *$ & 0,93 \\
\hline Afetividade & $34,11(7,8)$ & 38,00 & $28,10(9,7)$ & 30,00 & 47,50 & $0,033^{*}$ & 0,85 \\
\hline Adaptação & $22,44(3,8)$ & 24,00 & $20,24(5,47)$ & 22,00 & 68,00 & 0,228 & 0,05 \\
\hline
\end{tabular}

Mnd=mediana; $U=$ Mann-Whitney; $d^{*}=$ Tamanho de efeito dos resultados significativos realizado com o valor de $\mathrm{z}$. 
maior sobrecarga em comparação às mães sem estresse $(U=45,00, p<0,05, d=0,90)$.

No que diz respeito à percepção de suporte familiar medido pelo IPSF, $66,7 \%(n=6)$ das mulheres do GSE apresentaram escores de médio-alto a alto no IPSF-Total, enquanto no GCE são $52,4 \%(n=11)$. Nesse sentido, mães sem estresse $(M=78,00)$ apresentaram índices maiores no escore total do IPSF em comparação às mães com estresse $(M=62,00)$, com diferença também significativa $(U=48,00, p<0,05$, $d=0,84)$. Esse dado indica que mães do GSE tendem a perceber o suporte familiar recebido, de modo geral, como mais adequado em comparação às mães do GCE.

Ao estender as análises para os três fatores do IPSF (autonomia, afetividade e adaptação), houve diferenças significativas entre os grupos para autonomia $(U=45,00$, $p<0,05, d=0,93)$ e afetividade $(U=47,50, p<0,05$, $d=0,85)$. Mães do grupo sem estresse reportaram uma percepção maior de autonomia em comparação às mães com estresse, sugerindo que as primeiras percebem sua autonomia mais respeitada nas relações familiares que envolvem confiança, liberdade e privacidade entre os membros. O grupo sem estresse também teve uma mediana superior para o fator afetividade em relação às mães do grupo com estresse, indicando diferenças na percepção das relações afetivas entre seus familiares, como demonstrações verbais ou não verbais de carinho e conforto emocional.

\section{Discussão}

Este estudo teve por objetivo comparar mães de crianças com autismo, com e sem estresse, quanto à sobrecarga de cuidado, à autonomia da criança e à percepção de suporte familiar. Características sociodemográficas, percepção de estressores e sobrecarga de cuidado da mãe, suporte familiar, bem como autonomia da criança foram analisados.

Entre as participantes com estresse, houve o predomínio de sintomas psicológicos, sendo que estes envolvem desde uma agitação súbita a uma sensibilidade emocional ou irritabilidade excessivas, se comparado aos próprios parâmetros da pessoa (Lipp, 2000). Esses dados corroboram os achados da literatura que afirmam o alto índice de estresse em mães de crianças com TEA, tanto no Brasil como em vários países, destacando a amplitude do fenômeno e o impacto direto do TEA na qualidade de vida dessas mulheres (Fávero \& Santos, 2005; Pisula, 2011; Schmidt \& Bosa, 2007). Schmidt e Bosa (2007), utilizando ISSL (Lipp, 2000) em uma amostra de 30 mães, identificaram que $70 \%$ das mães apresentavam algum nível de estresse com predomínio em sintomas psicológicos, dados semelhantes aos encontrados neste estudo.

$\mathrm{Na}$ avaliação da autonomia, medida através do relato das mães sobre o desempenho funcional em ambos os grupos, a maioria das crianças apresentou escore abaixo do padrão normativo em relação ao autocuidado. Esses dados vão ao encontro de outros achados que avaliam as habilidades funcionais de crianças com TEA (Tomanik et al., 2004). Entretanto, Green e Carter (2014) ressaltam que o estágio do desenvolvimento em que criança se encontra, as dificuldades comportamentais e a gravidade dos sintomas estão envolvidos na relação entre o prejuízo funcional e o estresse materno. As autoras destacam, nesse sentido, que ganhos em habilidades da vida diária podem ser um fator de proteção para o bem-estar familiar (Green \& Carter, 2014).

Duas hipóteses foram pré-estabelecidas. A primeira hipótese, de que filhos de mães com estresse possuiriam maior prejuízo na autonomia, não foi corroborada pelos resultados. Na amostra, não foram encontradas diferenças significativas entre as características da criança de mães com e sem estresse, o que diverge dos achados da literatura. Vários autores têm apontado resultados opostos a esse, em que as características investigadas da criança (idade, autonomia e tempo de diagnóstico) tendem a apresentar correlação significativa com a presença de estresse (Dabrowska \& Pisula, 2010). Sifuentes e Bosa (2010), em seu estudo qualitativo, também identificaram que dificuldades dos filhos em realizar atividades da vida diária foram apontadas como um dos principais fatores de estresse das mães.

Nesse sentido, duas possíveis explicações são levantadas: (a) de que a amostra teve pouca variação entre grupos nas variáveis relacionadas autonomia e (b) que o instrumento não tenha sido sensível em avaliar as características do desempenho funcional em autocuidado, mobilidade e função social nesta amostra específica de crianças com TEA. Paicheco et al. (2010), em um estudo que comparou o desempenho funcional de amostras de 34 crianças com TEA e 27 crianças com deficiência intelectual, não encontraram diferenças significativas entre os grupos, concluindo que o mesmo instrumento não foi sensível para discriminar as duas condições, porém pontuaram que ele caberia como um guia de funcionalidade da criança em contexto mais clínico. Sobre esse aspecto, Bosa e Zanon (2016) apontam para as dificuldades em avaliação voltada para o público com TEA, visto o número limitado de instrumentos validados no Brasil, não só em pesquisas como para o uso clínico.

A segunda hipótese, de que haveria diferenças significativas entre a sobrecarga e a percepção do suporte 
familiar em mães com e sem estresse, foi confirmada. Os resultados encontrados evidenciaram a diferença entre grupos na percepção de sobrecarga. Mães de crianças com estresse apresentaram quase o dobro de sobrecarga, com um tamanho de efeito alto, sugerindo que, mesmo tratando-se de um estudo com tamanho amostral reduzido, o resultado foi significativo. Em direções opostas, a comparação entre grupos revelou também que a percepção de suporte familiar tende a ser maior em mães que não apresentam estresse, sendo mais evidente nos construtos de autonomia e afetividade. $\mathrm{Ou}$ seja, essas mães tendem a perceber que suas famílias respeitam suas decisões pessoais, a sua privacidade e liberdade diante da tomada de decisões, além de sentirem-nas como mais acolhedoras emocionalmente, pois demonstram e comunicam mais seus afetos e elogios entre os membros (Baptista, 2009).

Os dados reportados acima complementam os estudos que investigam a relação entre o estresse materno e as demandas de suporte que não são atendidas pela família. Kiami e Goodgold (2017), investigando estresse materno, estratégias de enfrentamento e as necessidades de suporte em 70 mães de crianças com TEA, identificaram que conforme aumentava as necessidades não atendidas, os índices de estresse materno também se elevavam. Dentre as citadas encontram-se: suporte financeiro, divisão de responsabilidades, ter ajuda no cuidado do filho, passar mais tempo sozinhas com o cônjuge, ter tempo para cuidar de suas próprias necessidades de saúde, entre outras. Dessa forma, o suporte familiar apresenta-se, conforme apontado por Baptista (2009), não só como um fator protetivo, mas como um atenuante diante de situações estressoras.

Smeha e Cezar (2011), em um estudo qualitativo, entrevistaram quatro mães de crianças com TEA, as quais relataram que a vivência da maternidade é uma experiência que envolve uma sobrecarga de responsabilidades e cuidado, sendo as relações familiares a principal fonte de auxílio nos momentos de adversidade. Pisula (2011) ressalta ainda, que os estudos vêm apontando justamente que o suporte social primário, ou seja, família nuclear ou pessoas próximas têm papel fundamental para o equilíbrio entre demandas de cuidado e distribuição de tarefas. Contudo, como reportado por Sifuentes e Bosa (2010), ainda é predominante a sobrecarga da mãe diante dos cuidados direcionados à criança, visto que a divisão de tarefas parentais não é igualitária.

Esses achados evidenciam que futuras intervenções podem auxiliar a adaptação da família com um membro com TEA, de modo a minimizar a sobrecarga e o estresse materno. Rolland (2016) defende que uma tarefa básica para as famílias, no contexto da doença crônica, é criar um significado para a situação de doença que preserve seu senso de competência e domínio. Deste modo, acredita-se que um processo familiar semelhante possa ocorrer no contexto dos transtornos desenvolvimentais. A perspectiva de Rolland (2016) enfatiza a necessidade de uma abordagem psicoeducacional preventiva que auxilie as famílias a anteverem desafios desenvolvimentais normativos associados à doença, de modo a maximizar um sentimento de controle e domínio por parte dos membros do sistema familiar.

Considera-se, ainda, a relevância de se oportunizar uma atenção ampliada junto às famílias de crianças com autismo como sistema mais amplo, compreendendo que o estresse materno pode afetar o sistema como um todo. Há uma influência mútua dos membros da família, portanto, eventos estressantes, condições ambientais e problemas de um membro (como aspectos socioemocionais e psicopatologias) afetam toda a família como uma unidade funcional, com reverberações para todos os membros e suas relações (Walsh, 2016b).

\section{Considerações finais}

Os desafios encontrados nas vivências de mulheres cujo filho apresenta o diagnóstico de TEA geram uma série de mudanças em suas vidas. O presente estudo pretendeu comparar mães de crianças com autismo, com e sem estresse, quanto à sobrecarga de cuidado, autonomia da criança e percepção de suporte familiar. As evidências apresentadas se somam à literatura da área ao apresentarem a relação significativa entre estresse e sobrecarga, bem como ao fato de mães sem estresse tenderem a perceber sua família como mais suportiva, o que parece neutralizar o efeito da sobrecarga. O controle das variáveis sociodemográficas e os tamanhos de efeito nos resultados significativos agregam maior valor aos resultados encontrados.

Contudo, ressalta-se que o estudo apresentou algumas limitações relevantes, como o tamanho amostral reduzido e a distribuição do número de participantes dos dois grupos (com e sem estresse) não equivalente. Assim, sugere-se para futuras pesquisas um número maior de participantes em ambos os grupos e instrumentos mais sensíveis para investigar as características das crianças e o estresse parental, além de incluir na investigação variáveis relacionadas à saúde mental e aspectos socioemocionais das mães. Foi observado, bem como reportado por outros autores (ver Brito \& Faro, 2016), que o instrumento ISSL, apesar de ser um dos mais utilizados no Brasil, não 
contempla as peculiaridades do estresse no contexto da parentalidade. Ressalta-se, portanto, nos estudos voltados para a parentalidade de crianças com TEA, a necessidade de instrumentos mais específicos para esse contexto. Os resultados encontrados também permitem traçar direcionamentos às políticas de saúde voltadas à atenção de familiares de crianças com TEA, indicando a importância do suporte familiar e a redução da sobrecarga do cuidador para a diminuição do estresse materno.

\section{Referências}

Amaral, K. C. (2013). Estresse e percepção de suporte familiar em mães de crianças com autismo (Dissertação de mestrado). Núcleo de Teoria e Pesquisa do Comportamento, UFPA, Belém. https://doi.org/10.25248/ reas28_2019

American Psychiatric Association (2013). Diagnostic and statistical manual of mental disorders (5 ${ }^{\text {th }}$ ed.). Washington, DC: Autor. (DOI INEXISTENTE)

Baptista, M. B. (2009). Inventário de percepção de suporte familiar (Vol. 1). São Paulo, SP: Vetor. (DOI INEXISTENTE)

Bosa, C. A., Sifuentes, M., \& Semensato, M. R. (2012). Coparentalidade e autismo: Contribuições teóricas e metodológicas. In Piccinini, C. A. \& Alvarenga, P. (Orgs.), Maternidade e Paternidade - A parentalidade em diferentes contextos (pp. 269-293). São Paulo, SP: Casa do Psicólogo. (DOI INEXISTENTE)

Bosa, C. A. \& Zanon, R. B. (2016). Psicodiagnóstico e transtorno do espectro autista. In C. S. Hutz, D. R. Bandeira, C. M. Trentini, \& J. S. Krug (Eds.), Psicodiagnóstico (pp. 308-322). Porto Alegre, RS: Artmed. (DOI INEXISTENTE)

Brito, A. \& Faro, A. (2016). Estresse parental: Revisão sistemática de estudos empíricos. Psicologia em Pesquisa, 10(1), 64-75. https://doi.org/10.24879/201600100010048

Cezar, P. K. \& Smeha, L. N. (2016). Repercussões do autismo no subsistema fraterno na perspectiva de irmãos adultos. Estudos de Psicologia, 33(1), 51-60. https://doi.org/10.1590/1982-02752016000100006

Dabrowska, A. \& Pisula, E. (2010). Parenting stress and coping styles in mothers and fathers of pre-school children with autism and Down syndrome. Journal of Intellectual Disability Research, 54(3), 266-280. https://doi.org/10.1111/j.1365-2788.2010.01258.x

Dillenburger, K., Keenan, M., Doherty, A., Byrne, T., \& Gallagher, S. (2010). Living with children diagnosed with autistic spectrum disorder: Parental and professional views. British Journal of Special Education, 37, 13-23. https://doi.org/10.1111/j.1467-8578.2010.00455.x

Estes, A., Munson, J., Dawson, D., Koehler, E., Zhou, X., \& Abbott, R. (2009). Parenting stress and psychological functioning among mothers of preschool children with autism and developmental delay. Autism, 13(4), 375-387. https://doi.org/10.1177/1362361309105658

Faro, A. \& Pereira, M. E. (2013). Estresse: Revisão narrativa da evolução conceitual, perspectivas teóricas e metodológicas. Psicologia, Saúde \& Doenças, 14(1), 78-100. https://doi.org/10.15309/13psd140106

Fávero, M. A. B. \& Santos, M. A. (2005). Autismo infantil e estresse familiar: Uma revisão sistemática da literatura. Psicologia: Reflexão e Crítica, 18(3), 358-369. https://doi.org/10.1590/s0102-79722005000300010

Fodstad, J. C. \& Matson, J. L. (2008). A comparison of feeding and mealtime problems in adults with intellectual disabilities with and without autism. Journal of Developmental and Physical Disabilities, 20(6), 541-550. doi:10.1007/s10882-008-9116-6 https://doi.org/10.1007/s10882-008-9116-6

Fombonne, E. (2009). Epidemiology of pervasive developmental disorders. Pediatric Research, 65(6), 591-598. https://doi.org/10.1203/PDR.0b013e31819e7203

García-Lopez, C., Sarriá, E., \& Pozo, P. (2016). Multilevel approach to gender differences in adaptation in father-mother dyads parenting individuals with Autism Spectrum Disorder. Research in Autism Spectrum Disorders, 28, 7-16. https://doi.org/10.1016/j.rasd.2016.04.003

Gomes, V. F. \& Bosa, C. (2004). Estresse e relações familiares na perspectiva de irmãos de indivíduos com transtornos globais do desenvolvimento. Estudos de Psicologia, 9(3), 553-561. https://doi.org/10.1590/s1413$294 \times 2004000300018$ 
Gomes, P. T. M., Lima, L. H. L., Bueno, M. K. G., Araújo, L. A., \& Souza, N. M. (2015). Autism in Brazil: A systematic review of family challenges and coping strategies. Jornal de Pediatria (Rio J), 91(2), 111-121. https://doi.org/10.1016/j.jpedp.2015.01.005

Gorlin, J. B., McAlpine, C. P., Garwick, A., \& Wieling, E. (2016). Severe childhood autism: The family lived experience. Journal of Pediatric Nursing, 31(6), 580-597. https://doi.org/10.1016/j.pedn.2016.09.002

Green, S. A. \& Carter, A. S. (2014). Predictors and course of daily living skills development in toddlers with Autism Spectrum Disorders. Journal of Autism and Developmental Disorders, 20, 1-8. https://doi.org/10.1007/ s10803-011-1275-0

Haley, S. M., Coster, W. J., Ludlow, L. H., Haltiwanger, J. T., \& Andrellos, P. J. (1992). Pediatric evaluation of disability inventory (PEDI): Development, standarlization and administration manual (versão 1). Boston, MA: Trustees of Boston University. https://doi.org/10.1037/t08316-000

Hartley, S. L. \& Schultz, H. M. (2015). Support needs of fathers and mothers of children and adolescents with Autism Spectrum Disorder. Journal of Autism and Developmental Disorders, 45(6), 1636-1648. https://doi. org/10.1007/s10803-014-2318-0

Hastings, R., Kovshoff, H., Ward, N., Espinosa, F., Brown, T., \& Remington, B. (2005). Systems analysis of stress and positive perceptions in mothers and fathers of pre-school children with autism. Journal of Autism and Developmental Disorders, 35(5), 645-654. https://doi.org/10.1007/s10803-005-0007-8

Hill, J., Fonagy, P., Safier, E., \& Sargent, J. (2003). The ecology of attachment in the family. Family Process, 42 , 205-221. https://doi.org/10.1111/j.1545-5300.2003.42202.x

Kiami, S. R. \& Goodgold, S. (2017). Support needs and coping strategies as predictors of stress level among mothers of children with autism spectrum disorder. Autism Research and Treatment, v. 2017, ID 8685950, 1-10. https://doi.org/10.1155/2017/8685950

Lazarus, R. S. \& Folkman, S. (1984). Stress, appraisal, and coping. New York: Springer.

Lipp, M. E. N. (2000). Manual do inventário de sintomas de stress para adultos de Lipp. São Paulo, SP: Casa do Psicólogo. (DOI INEXISTENTE)

Macedo, E. C., Silva, L. R., Paiva, M. S., \& Ramos, M. N. P. (2015). Sobrecarga e qualidade de vida de mães de crianças e adolescentes com doença crônica: Revisão integrativa. Revista Latino-Americana de Enfermagem, 23(4), 769-777. https://doi.org/10.1590/s0104-11692005000500010

Mancini, M. C. (2005). Inventário de avaliação pediátrica de incapacidade (PEDI): Manual da versão brasileira. Belo Horizonte, MG: Editora UFMG. (DOI INEXISTENTE)

Meimes, M. A., Saldanha, H. C., \& Bosa, C. A. (2015). Adaptação materna ao transtorno do espectro autismo: Relações entre crenças, sentimentos e fatores psicossociais. Psico, 46(4), 412-422. https://doi.org/10.15448/19808623.2015.4.18480

Misquiatti, A. R. N, Brito, M. C., Ferreira, F. T. S., \& Assumpção Junior, F. B. (2015). Sobrecarga familiar e crianças com transtornos do espectro do autismo: Perspectiva dos cuidadores. Revista CEFAC, 17(1), 192-200. https://doi.org/10.1590/1982-0216201520413

Paicheco, R., Di Matteo, J., Cucolicchio, S., Gomes, C., Simone, M. F., \& Assumpção Jr., F. B. (2010). Inventário de Avaliação Pediátrica de Incapacidade (PEDI): Aplicabilidade no diagnóstico de transtorno invasivo do desenvolvimento e retardo mental. Revista Medicina de Reabilitação, 29(1), 9-12. (DOI INEXISTENTE)

Pereira, M. G. \& Soares, A. J. (2011). Sobrecarga em cuidadores informais de dependentes de substâncias: Adaptação do Caregiver Reaction Assessment (CRA). Psicologia, Saúde \& Doenças, 12(2), 304-328. https://doi.org/10.15309/11psd120211

Phetrasuwan, S. \& Miles, M. S. (2009). Parenting stress in mothers of children with autism spectrum disorders. Journal for Specialists in Pediatric Nursing, 14(3), 157-165. https://doi.org/10.1111/j.1744-6155.2009.00188.x

Pisula, E. (2011). Parenting stress in mothers and fathers of children with autism spectrum disorders. In Mohammadi, M. (Ed.). A comprehensive book on autism spectrum disorders (pp. 87-105). Recuperado de http://www.intechopen. com. https://doi.org/10.5772/18507

Pozo, P. \& Sarriá, E. (2014). Prediction of stress in mothers of children with autism spectrum disorders. Spanish Journal of Psychology, 17, 1-12. https://doi.org/10.1017/sjp.2014.6 
Rolland, J. S. (2001). Doença crônica e o ciclo de vida familiar. In Carter, B., McGoldrick, M. (Eds.). As mudanças no ciclo de vida familiar: uma estrutura para a terapia familiar (pp. 373-392). Porto Alegre, RS: Artmed. https://doi.org/10.17771/pucrio.acad.11214

Rolland, J. S. (2016). Enfrentando os desafios familiares em doenças graves e incapacidade. In Walsh, F., Processos normativos da família: Diversidade e complexidade (Mallmann, S. M., Mosmann, C. P., Wagner, A., Trad.). (pp. 452-482). Porto Alegre, RS: Artmed. (DOI INEXISTENTE)

Schmidt, C. \& Bosa, C. A. (2003). A investigação do impacto do autismo na família: Revisão crítica da literatura e proposta de um novo modelo. Interação em Psicologia, 7(2), 111-120. https://doi.org/10.5380/psi.v7i2.3229

Schmidt, C., Dell'Aglio, D. D., \& Bosa, C. A. (2007). Estratégias de coping de mães de portadores de autismo: Lidando com dificuldades e com a emoção. Psicologia: Reflexão e Crítica, 20(1), 124-131. https://doi.org/10.1590/ s0102-79722007000100016

Sifuentes, M. \& Bosa, C. A. (2010). Criando pré-escolares com autismo: Características e desafios da coparentalidade. Psicologia em Estudo, 15(3), 477-485. https://doi.org/10.1590/S1413-73722010000300005

Smeha, L. N. \& Cezar, P. K. (2011). A vivência da maternidade de mães de crianças com autismo. Psicologia em Estudo, 16(1), 43-50. https://doi.org/10.1590/s1413-73722011000100006

Taub, A., Adreoli, S. B., \& Bertolucci, P. H. (2004). Dementia caregiver burden: Reliability of the Brazilian version of the Zarit caregiver burden interview. Cadernos de Saúde Pública, 20(2), 372-376. https://doi.org/10.1590/s0102$311 \times 2004000200004$

Tomanik, S., Harris, G. E., \& Hawkins, J. (2004). The relationship between behaviours exhibited by children with autism and maternal stress. Journal of Intellectual \& Developmental Disability, 29(1), 16-26. https://doi.org/ $10.1080 / 13668250410001662892$

Walsh, F. (2016a). Resiliência familiar In Walsh, F., Processos normativos da família: Diversidade e complexidade (Mallmann, S. M., Mosmann, C. P., Wagner, A., Trad.) (pp. 28-54). Porto Alegre, RS: Artmed. (DOI INEXISTENTE)

Walsh, F. (2016b). Visões clínicas de normalidade, saúde e disfunção familiar. In Walsh, F., Processos normativos da familia: Diversidade e complexidade (Mallmann, S. M., Mosmann, C. P., Wagner, A., Trad.) (pp. 28-54). Porto Alegre, RS: Artmed. (DOI INEXISTENTE)

Zaidman-Zait, A., Mirenda, P., Duku, E., Vaillancourt, T., Smith, I. M., Szatmari, P., ..., \& Thompson, A. (2017). Impact of personal and social resources on parenting stress in mothers of children with autism spectrum disorder. Autism, 21(2), 155-166. https://doi.org/10.1177/1362361316633033

Zarit, S. H., Reever, K. E., \& Back-Peterson, J. (1980). Relatives of the impaired elderly: Correlates of feelings of burden. The Gerontologist, 20, 649-655. https://doi.org/10.1093/geront/20.6.649

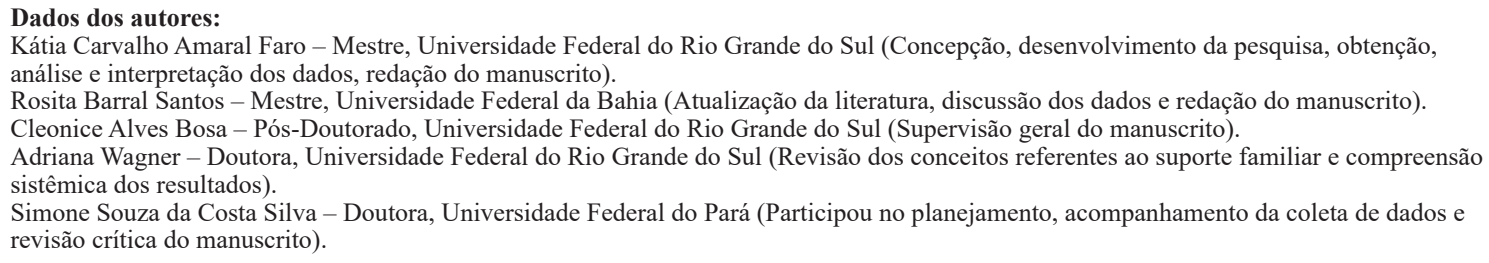

Kátia Carvalho Amaral Faro - Mestre, Universidade Federal do Rio Grande do Sul (Concepção, desenvolvimento da pesquisa, obtenção, análise e interpretação dos dados, redação do manuscrito).

Rosita Barral Santos - Mestre, Universidade Federal da Bahia (Atualização da literatura, discussão dos dados e redação do manuscrito). Cleonice Alves Bosa - Pós-Doutorado, Universidade Federal do Rio Grande do Sul (Supervisão geral do manuscrito)

Adriana Wagner - Doutora, Universidade Federal do Rio Grande do Sul (Revisão dos conceitos referentes ao suporte familiar e compreensão sistêmica dos resultados).

Simone Souza da Costa Silva - Doutora, Universidade Federal do Pará (Participou no planejamento, acompanhamento da coleta de dados e revisão crítica do manuscrito).

\section{Endereco para correspondência:}

Kátia Carvalho Amaral Faro

Universidade Federal do Rio Grande do Sul

Rua Ramiro Barcelos, 2.600 - Santana

90035-003, Porto Alegre, RS, Brasil

E-mail: katia.c.psi@gmail.com

Recebido em: 08/03/2018.

Aceito em: 06/12/2018.

Publicado em: 05/08/2019. 